\title{
Discovering the genetic contribution to cardiovascular diseases in patients affected by autoimmune diseases
}

\author{
Cinzia Ciccacci \\ Department of Biomedicine and Prevention, Section of Genetics, School of Medicine, University of Rome Tor Vergata, Rome, Italy \\ Correspondence to: Cinzia Ciccacci. Department of Biomedicine and Prevention, Section of Genetics, School of Medicine, University of Rome Tor \\ Vergata, Rome, Italy. Email: cinziaciccacci@libero.it. \\ Comment on: Leonard D, Svenungsson E, Dahlqvist J, et al. Novel gene variants associated with cardiovascular disease in systemic lupus erythematosus \\ and rheumatoid arthritis. Ann Rheum Dis 2018;77:1063-9.
}

Submitted Sep 21, 2018. Accepted for publication Sep 29, 2018.

doi: $10.21037 / \mathrm{atm} .2018 .09 .67$

View this article at: http://dx.doi.org/10.21037/atm.2018.09.67

Autoimmune diseases (AD) are complex diseases characterised by alteration of the immune system and production of autoantibodies. Their pathogenesis is multifactorial since both the environment and genetic factors are involved. They present a large variability in the presentation of symptoms, the course of diseases and the clinical complications. Although, some AD can present common characteristics, often each disease presents typical symptoms and manifestations and disease severity could be very different. The identification of genetic loci associated with these diseases is a crucial step to define risk genetic profiles and to stratify patients both for disease course and for the therapeutic approach. There is an abundant literature about the genetic risk loci for $\mathrm{AD}$ and, in particular, in last decade the use of genome wide association studies (GWAs) has permitted the discovery of hundreds of risk variants in several $\mathrm{AD}$ (1). Unfortunately, often these studies are poor in reporting associations between genetic variants and clinical phenotypes. Indeed, GWAs require large numbers of analysed samples and, to reach such numbers, patients were collected in different centres and in different countries, with a great difficulty to collect clinical data in homogenous way. Therefore, there is still a gap in understanding the role of genetics in the development of particular manifestations of the diseases.

Recently, Leonard et al. (2) have published an interesting paper focused on the genetics of cardiovascular diseases (CVD) in patients affected by $\mathrm{AD}$, in particular systemic lupus erythematosus (SLE) and rheumatoid arthritis (RA). This topic is of large interest since CVD is one of the causes of mortality and morbidity both in SLE and RA (3). The authors have investigated a large sample of SLE patients from Swedish and a replication cohort from USA and a second large sample of Sweden RA patients.

In detail, they performed a large genotyping by using an Illumina Immunochip, investigating 137,213 SNPs in the SLE discovery cohort. Following, they searched for the associations between SNPs and CVD risk in general, and for a more specific risk of ischaemic stroke, myocardial infarction (MI), angina, transient ischaemic attack (TIA) pulmonary embolism and deep vein thrombosis. Three loci (IL-19, IL-7R and SRP54/AS1 gene) were associated with CVD both in the discovery and in the replication cohort. Specifically, IL-19 SNPs were associated with stroke/MI, while the SRP54-AS1 and IL-7R SNPs were associated with stroke/TIA. The association between IL-19 and stroke/MI was confirmed also in the RA cohort. In addition, in RA patients, IL-19 SNPs showed association also with stroke/TIA. These associations remained significant also after correction for classical cardiovascular risk factors. Through functional studies the Authors hypothesised, that IL-19 risk locus can regulate IL-10 expression, enhancing the production of aCL (a well-known prothrombotic autoantibody) by B cells. More complicated is to find a role for the SRP54/AS1 gene, but the Authors observed that it could potentially regulate FAM177A1, a gene expressed in the artery wall. On the contrary, functional studies did not reveal any evidence to explain the association with IL-7R.

The strength of the study of Leonard et al. is firstly the large sample size investigated and secondly the well-defined 
cardiovascular phenotypes. Indeed, the majority of published genetic association studies on SLE and RA were not able to investigate the contribution of genetic loci in subgroups of patients with specific complications, such as cardiovascular diseases. Moreover, at the light of their results, there at least two aspects that merit to be highlighted. Firstly, the variant alleles of associated SNPs do not confer a risk in the general CVD population, suggesting the involvement of different mechanisms. This is in agreement with the observation that SLE patients have a 3-fold risk to develop subclinical atherosclerosis, an important factor to predict cardiovascular events, independently by traditional CVD risk factors and treatments (4). Secondly, there are no evidence that CVD appear in patients with more severe forms of SLE or RA. It is well-known that atherosclerosis derives from a chronic inflammation and that specific immune alterations could play a pivotal role since in the first stage of atherosclerotic process (5). Therefore, considering the inflammatory status of $\mathrm{AD}$ and the alteration of immune pathways, it is possible more than a link between autoimmunity and cardiovascular events.

The associations with IL-19 and SRP54/AS1 should be investigated also in other ADs, different from SLE and RA, to verify if these variants are common risk factors for different diseases or specific for SLE and RA. The heterogeneity of the phenotypic spectrum in patients with $\mathrm{ADs}$ could be due to different genetic contributions that could lead to diverse immune-pathological consequences. Moreover, it should be interesting to investigate these SNPs in non-European populations, particularly in African populations where the risk for SLE patients to develop cardiovascular diseases is double compared to non-African ones (6).

Moreover, it is worth to consider that some comorbidities, such as cardiovascular diseases in SLE or RA, can be due not only to diseases itself, but also to treatments and to the co-presence of other AD (7). Another aspect to consider is the epigenetics that includes DNA methylation, histone modifications and microRNA activity (miRNA). Epigenetics represents a link between genomics and environmental factors and regulates gene expression independently from DNA variation. It is possible that disease itself or treatments can induce some epigenetics changes, for example in methylation status or in miRNA profiles, acting on molecules involved with atherosclerosis process and endothelial cells activity. In this context, it is evident that some microRNAs could control hundreds of genes and consequently be involved in several pathways contemporary. For example, mir-124, mir-155, mir-146 are well known to be altered both in autoimmunity and in atherosclerosis. It is suggested that ADs patients with thrombotic evidences exhibit a different miRNA expression profile due to the regulation by specific immune factors, such as the autoantibodies (8).

Given the complexity of the interaction between autoimmunity and cardiovascular events, further studies focused on sub-phenotypes are necessary to improve our knowledge and for the identification of specific predictive genetic biomarkers. New genomics approaches such as Next Generation Sequencing searching for rare predisposing alleles, epigenetics studies including methylation status and microRNA expression profiles will permit, in the next future, to better understand the genetic basis of CVD in patients affected by ADs.

\section{Acknowledgements}

None.

\section{Footnote}

Conflicts of Interest: The author has no conflicts of interest to declare.

\section{References}

1. Lessard CJ, Ice JA, Adrianto I, et al. The genomics of autoimmune disease in the era of genome-wide association studies and beyond. Autoimmun Rev 2012;11:267-75.

2. Leonard D, Svenungsson E, Dahlqvist J, et al. Novel gene variants associated with cardiovascular disease in systemic lupus erythematosus and rheumatoid arthritis. Ann Rheum Dis 2018;77:1063-9.

3. Durante A, Bronzato $S$. The increased cardiovascular risk in patients affected by autoimmune diseases: review of the various manifestations. J Clin Med Res 2015;7:379-84.

4. Kravvariti E, Konstantonis G, Sfikakis PP, et al. Progression of subclinical atherosclerosis in systemic lupus erythematosus versus rheumatoid arthritis: the impact of low disease activity. Rheumatology (Oxford) 2018. [Epub ahead of print].

5. Libby P. Inflammation in atherosclerosis. Nature 2002;420:868-74.

6. Alenghat FJ. The Prevalence of Atherosclerosis in Those with Inflammatory Connective Tissue Disease by Race, Age, and Traditional Risk Factors. Sci Rep 2016;6:20303. 
7. Teixeira V, Tam LS. Novel Insights in Systemic Lupus Erythematosus and Atherosclerosis. Front Med (Lausanne) 2018;4:262.

8. Pérez-Sánchez C, Aguirre MA, Ruiz-Limón P, et

Cite this article as: Ciccacci C. Discovering the genetic contribution to cardiovascular diseases in patients affected by autoimmune diseases. Ann Transl Med 2018;6(Suppl 1):S44. doi: 10.21037/atm.2018.09.67 al. Atherothrombosis-associated microRNAs in Antiphospholipid syndrome and Systemic Lupus Erythematosus patients. Sci Rep 2016;6:31375. 